\title{
Strong unitary violations in the extra dimensional SM and the equivalence between Goldstone and longitudinal gauge bosons.
}

\author{
José R. Pelaez ${ }^{* \dagger}$ \\ Dept. Física Teórica II. Universidad Complutense, Spain \\ E-mail: jrpelaez@fis.ucm.es
}

\section{S. De Curtis and D. Dominici}

INFN Sezione di Firenze and Dip. di Fisica Universitá degli Studi, Firenze.

E-mail: decurtis@fi.infn.it,dominici@fi.infn.it

\begin{abstract}
Tree level unitarity violations of extra dimensional extensions of the Standard Model may become much stronger when the scalar sector is included in the bulk. This effect occurs when the couplings are not suppressed for larger Kaluza-Klein levels, and could have relevant consequences for the phenomenology of the next generation of colliders. We briefly review our formalism to obtain more stringent unitarity bounds when KK modes are present, as well as the generalization to extra dimensions of the Equivalence Theorem between Goldstone bosons and longitudinal gauge bosons.
\end{abstract}

International Europhysics Conference on High Energy Physics

July 21 st - 27th 2005

Lisboa, Portugal

\footnotetext{
${ }^{*}$ Speaker.

${ }^{\dagger}$ Work partially supported by an INFN-CICYT collaboration project.
} 


\section{Introduction.}

Violations of tree level unitarity are an indication of a perturbation theory breakdown and that the interaction has become strong. As it is well known, this happens in the Standard Model (SM) for Higgs masses of the order of $1 \mathrm{TeV}$. Let us now recall how this bound was obtained [1]. First, we need the definition of partial waves of angular momentum $J$ for two-body scattering, and the familiar unitarity condition they satisfy in the elastic regime, when only one state $\alpha$ is available:

$t_{\alpha \beta}^{J}(s) \equiv \frac{1}{32 \pi} \int_{-1}^{1} d(\cos \theta) T_{\alpha \beta}(s, t, u) P_{J}(\cos \theta), \quad \stackrel{\text { elastic }}{\longrightarrow} \operatorname{Im} t_{\alpha \alpha}^{J}=\sigma_{\alpha}\left|t_{\alpha \alpha}^{J}\right|^{2}, \quad \sigma_{\alpha}=2 q_{\alpha} / \sqrt{s}$

$q_{\alpha}$ being the CM momenta. Note that in the $s \rightarrow \infty$ limit, $\sigma_{\alpha} \rightarrow 1$ and unitarity implies $\left|t_{\alpha \alpha}\right|^{J} \leq 1$. When more states, $\beta$, are accessible, we form a matrix $T_{\alpha \beta}^{J}$ and unitarity requires its eigenvalues to be smaller than one. For example, in the SM we have tree level couplings between $\alpha=H H / \sqrt{2}$, $\beta=Z_{L} Z_{L} / \sqrt{2}, \gamma=W_{L}^{+} W_{L}^{-}$. Thus, in the $s \rightarrow \infty$ limit, $T_{\alpha \beta}^{J=0}$ reads

$$
T^{J=0}=\frac{G_{F} m_{h}^{2}}{4 \pi \sqrt{2}}\left(\begin{array}{ccc}
1 & 1 / \sqrt{8} & 1 / \sqrt{8} \\
1 / \sqrt{8} & 3 / 4 & 1 / 4 \\
1 / \sqrt{8} & 1 / 4 & 3 / 4
\end{array}\right) \stackrel{\text { Eigenvalues }}{\longrightarrow} \frac{G_{F} m_{h}^{2}}{8 \pi \sqrt{2}}(3,1,1) \leq 1 \stackrel{\text { Unitarity }}{\longrightarrow} m_{h}^{2} \leq \frac{8 \pi \sqrt{2}}{3 G_{F}} .
$$

\section{Unitarity conditions including Kaluza Klein excitations.}

In a recent work [2], we have shown that unitarity bounds can become more stringent in extra dimensional extensions of the SM. In fact, any additional Kaluza-Klein (KK) state, $\beta$, coupled to the SM states above, $\alpha$, always provide additional contributions to the unitarity bound, i.e.

$$
\mathrm{Unit}_{\alpha \rightarrow \alpha} \equiv \sigma_{\alpha}\left|t_{\alpha \alpha}^{J}\right|+\frac{1}{\left|t_{\alpha \alpha}^{J}\right|} \sum_{\beta \neq \alpha} \sigma_{\beta}\left|t_{\alpha \beta}^{J}\right|^{2} \leq 1
$$

Thus, tree level unitarity violations occur for smaller $m_{h}$ and lower $s$ than in the SM. Of course, in models where KK states do not couple to Higgs or longitudinal gauge bosons, or where their tree level couplings are sufficiently suppressed, Eq.(2.1) still holds, but the new terms may be negligible. However we have shown that this effect can be rather large when Higgs fields extend in the bulk and their KK modes have the same couplings as the zero mode. This is the case, for instance, of universal extra dimensions [3]. Here we will briefly review how, with compactification scales of the order of $1 / R \simeq 0.5-3 \mathrm{TeV}$, this effect could lead to a strongly interacting regime within the reach of the LHC. In order to do so, we first review a useful tool to calculate longitudinal gauge boson amplitudes, and its extension to extra dimensions.

\section{The Equivalence Theorem in extra dimensions.}

In renormalizable $R_{\xi}$ gauges, the gauge fixing function $f_{a}=\partial_{\mu} V^{\mu a}-\xi M G^{V a}$, which acts like a delta function in field space, implies $p_{\mu} V^{\mu a}=\xi M G^{V a}$. Since $\varepsilon_{L \mu}=p_{\mu} / M+O(M / \sqrt{s})$, then $V_{L} \equiv \varepsilon_{L \mu} V^{\mu} \simeq G^{V}$ and, up to $O(M / \sqrt{s})$, the Equivalence Theorem [4] allows us to calculate amplitudes for longitudinal gauge bosons by replacing them by their associated Goldstone bosons. 
We have used an extra dimensional generalization [5] with 5D gauge bosons and Higgs fields in the bulk, on the brane, or both. The novelty is that mass matrices are different now for $V_{L}$ and $G^{V}$ and are non diagonal, mixing the Goldstone boson KK modes $G_{(n)}^{V}$ with the $V_{(n)}^{5}$ (the latter give masses to the $\mathrm{KK} V_{L}$ in the unbroken case [6]). If the Higgs is on the brane there is also KK level mixing. We thus had to perform a rotation to find the gauge boson mass eigenstates and a different one to diagonalize the Goldstone boson "mass" matrix $M_{G}^{2}$. It was not trivial to find that the mixing term from the gauge fixing function, proportional to $M_{G}$ and not to $M_{G}^{2}$, becomes diagonal under these simultaneous rotations. This implies that there is a complicated combination of Goldstone bosons and $V_{(n)}^{5}$ KK modes, that is a "mass" eigenstate, which is related ("eaten") by their associated longitudinal gauge boson mass eigenstate, so that, as usual,

$$
T\left(\hat{V}_{L(m)}^{\mu}, \hat{V}_{L(n)}^{\mu}, \ldots\right) \simeq C^{(m)} C^{(n)} \ldots T\left(\hat{G}_{(m)}^{V}, \hat{G}_{(n)}^{V}, \ldots\right)+O\left(M_{k} / E\right)
$$

$M_{k}$ being the largest $m_{V(m)}$ mass, and $C^{(i)}=1+$ "electroweak corrections"[4]. Since the $\hat{G}^{V}$ are scalars, in the Landau gauge the calculations are considerably simplified. The $a_{(n)}$ combinations of $G^{V}$ and $V^{5}$ not "eaten" by the $V_{L}$, remain massive with $m_{a(n)}^{2}=m_{h(0)}^{2}+n^{2} / R^{2}$.

\section{An example of strong unitarity violations with scalars in the bulk.}

Let us consider a minimal 5D extension of the SM compactified on the segment $S^{1} / Z_{2}$, of length $\pi R$, in which the $S U(2)_{L}$ and $U(1)_{Y}$ gauge and Higgs fields propagate in the bulk. It is straightforward to write the Lagrangian and tree level partial waves [2] of the state $\alpha=W_{L(0)}^{+} W_{L(0)}^{-}$ scattering into itself, as well as into $\beta=h_{(0)} h_{(0)} / \sqrt{2}, Z_{L(0)} Z_{L(0)} / \sqrt{2}, h_{(n)} h_{(n)} / \sqrt{2}, a_{(n)}^{3} a_{(n)}^{3} / \sqrt{2}$ and $a_{(n)}^{+} a_{(n)}^{-}$, whose interactions are not suppressed by any power of $R$ or EW couplings.

In Figure 1 we plot the left hand side of Eq.(2.1), for $1 / R=0.5 \mathrm{TeV}$ and $3 \mathrm{TeV}$, and we see that tree level unitarity violations occur for smaller values of $m_{h(0)}$ and $\sqrt{s}$ than in the SM.
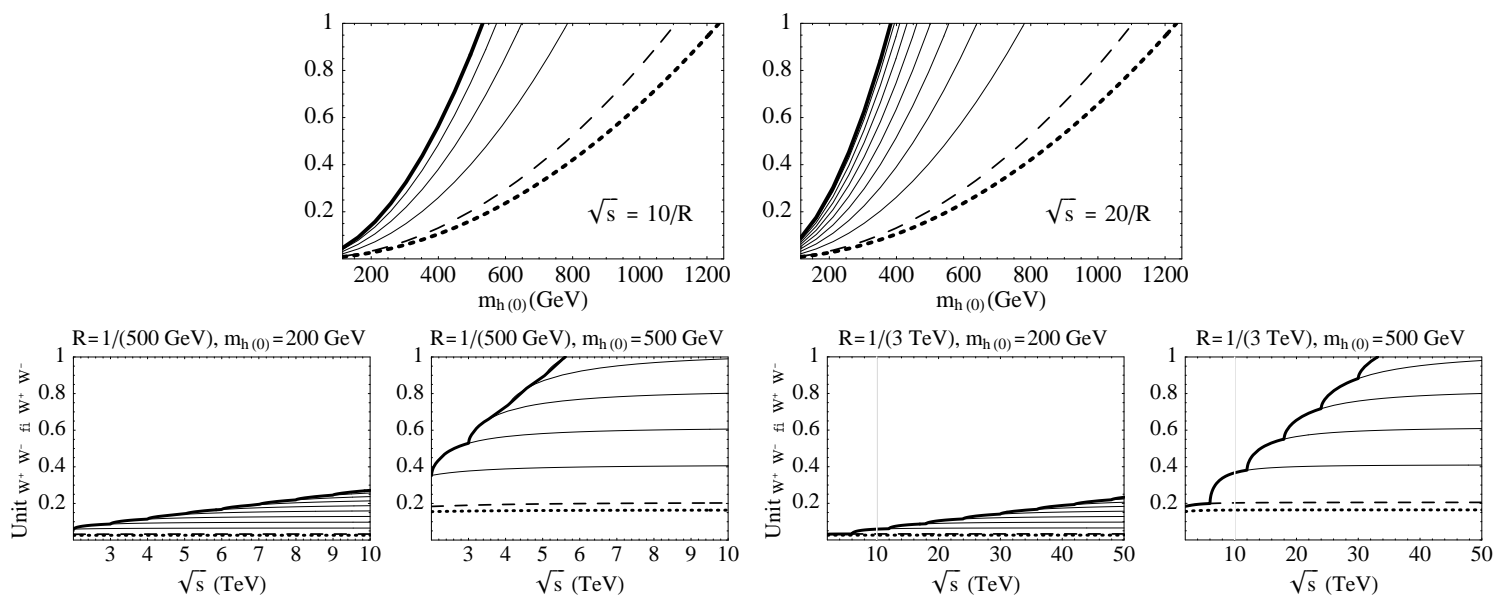

Figure 1: The unitarity bound, Eq.(2.1) is violated above 1. The dotted line is the SM bound using only $W_{L(0)}^{+} W_{L(0)}^{-}$elastic scattering, whereas the dashed line includes also the $h_{(0)} h_{(0)}, Z_{L(0)} Z_{L(0)}$. The continuous lines are the contributions of the KK excitations, which, in total, yield the thick continuous line.

In Figure 2 we plot in the $\left(\sqrt{s}, m_{h(0)}\right)$ plane the regions where tree level unitarity is violated (white). Since we have only used two-body states to derive Eq.(2.1), and other, many body, states 
should also contribute positively to the sum, we have also plotted in gray the area where Eq.(2.1) is larger than 0.5 . In these two regions the electroweak symmetry breaking sector will most likely become strongly interacting. Finally, we can also see how the more dimensions are added $(D=$ $6,7 \ldots)$, the stronger tree level unitarity violations become. Finally, let us remark that this effect could be accessible at the next generation of colliders, like LHC.
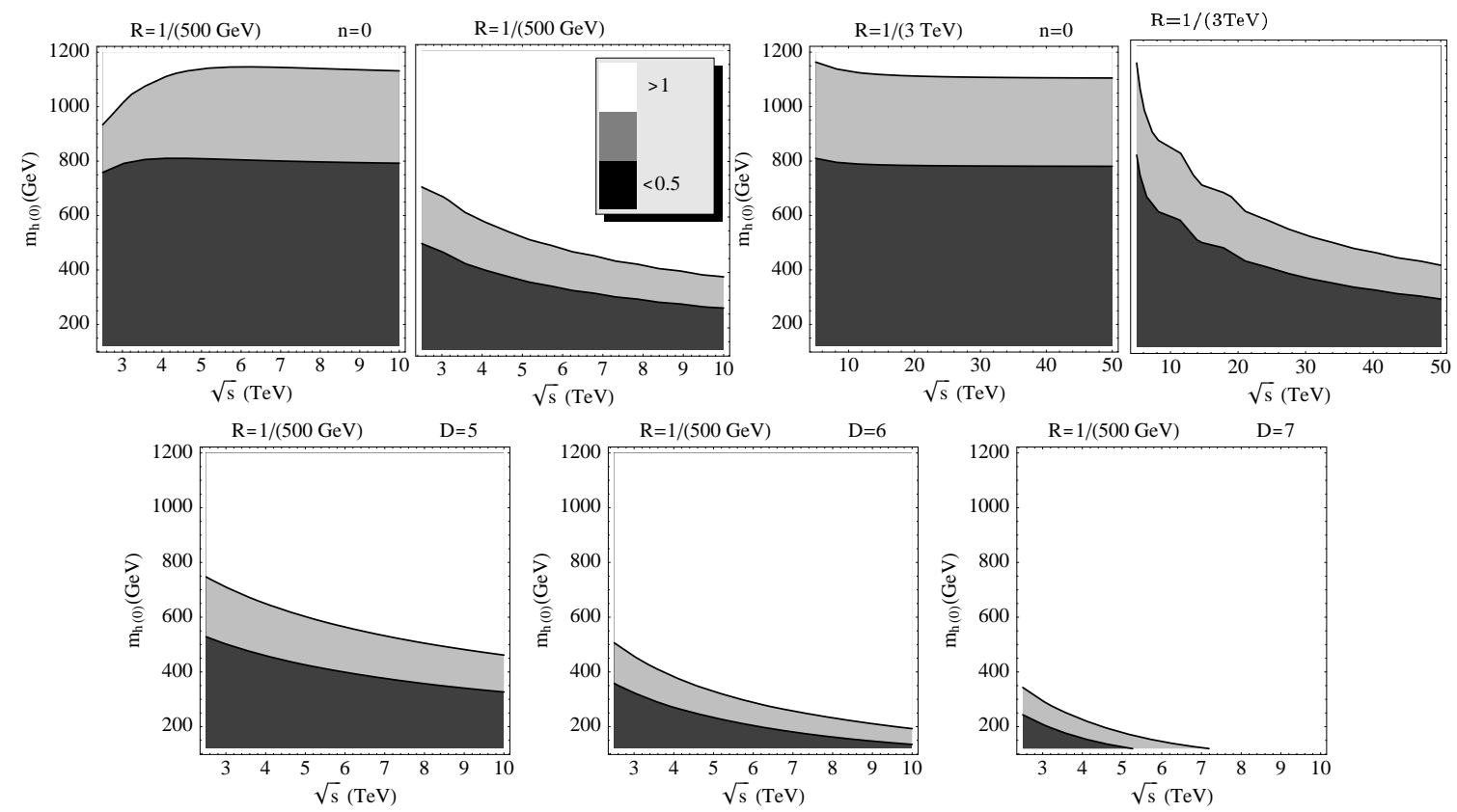

Figure 2: Tree level unitarity violation (white) and possibly strongly interacting regions (gray) for different values of $R, \sqrt{s}, m_{h(0)}$ and dimensions $D$, for models with unsuppressed KK scalar couplings.

\section{References}

[1] B. W. Lee, C. Quigg and H. B. Thacker, Phys. Rev. D 16 (1977) 1519.

[2] S. De Curtis, D. Dominici and J. R. Pelaez, Phys. Rev. D 67, 076010 (2003)

[3] R. Barbieri, L. J. Hall and Y. Nomura, Phys. Rev. D 63 (2001) 105007; T. Appelquist, H. C. Cheng and B. A. Dobrescu, Phys. Rev. D 64 (2001) 035002.T. Appelquist and H. U. Yee, Phys. Rev. D 67, $055002(2003)$

[4] J. M. Cornwall, D. N. Levin and G. Tiktopoulos, Phys. Rev. D10 (1974) 1145; C. E. Vayonakis, Lett. Nuovo Cim. 17 (1976) 383; M. S. Chanowitz and M. K. Gaillard Nucl. Phys. B261 (1985) 379; G. K. Gounaris, R. Kogerler and H. Neufeld, Phys. Rev. D34 (1986) 3257; Y. P. Yao and C. P. Yuan, Phys. Rev. D38 (1988) 2237; J. Bagger and C. Schmidt, Phys. Rev. D41 (1990) 264; H.J. He, Y. P. Kuang, and X. Li Phys. Rev. Lett. 69 (1992) 2619.

[5] S. De Curtis, D. Dominici and J. R. Pelaez, Phys. Lett. B 554, 164 (2003)

[6] R. S. Chivukula, D. A. Dicus and H. J. He, Phys. Lett. B 525 (2002) 175; R. S. Chivukula and H. J. He, Phys. Lett. B 532 (2002) 121. 